\title{
INSPIRATORY MUSCLE TRAINING ON FUNCTIONAL CAPACITY AFTER CORONARY ARTERY BYPASS GRAFTING IN PATIENTS WITH HIGH RISK OF PULMONARY COMPLICATION: CLINICAL TRIAL
}

\author{
André Luiz Lisboa Cordeiro ${ }^{1}$, Bianca Carvalho ${ }^{1}$, Eduarda Silva ${ }^{1}$, Natália Santos ${ }^{1}$, André \\ Guimarães $^{2}$, and Jefferson Petto ${ }^{3}$ \\ ${ }^{1}$ Faculdade Nobre \\ ${ }^{2}$ Instituto Nobre de Cardiologia \\ ${ }^{3}$ Escola Bahiana de Medicina e Saude Publica
}

June 24, 2020

\begin{abstract}
Objective: To assess the impact of IMT on the functional capacity of patients undergoing CABG with a high risk of PPC. Design: This is a randomized clinical trial. Setting: Instituto Nobre de Cardiologia (INCARDIO), Feira de Santana - Bahia. Subjects: In total, 29 patients were selected at high risk of pulmonary complications. Intervention: Patients were divided into two groups at risk of CPP: intervention group (IM) submitted to IMT and control group (CG) who received standard care. Considered a high risk patient, age over 60 years, diabetic, smoker and body mass index above $27 \mathrm{~kg} / \mathrm{m} 2$. All were submitted to preoperative evaluation and hospital discharge for maximum inspiratory pressure (MIP), maximum expiratory pressure (MEP) and six-minute walk test (6MWT). Results: 29 individuals were evaluated, 19 of whom were female $(65 \%)$ and the mean age was $67 \pm 4$ years. The IG showed MIP before of $103 \pm 15$ and the CG $105 \pm 17 \mathrm{cmH} 2 \mathrm{O}(\mathrm{p}=0.35$ ), at discharge the IG had $80 \pm 14$ and the CG $75 \pm 15 \mathrm{cmH} 2 \mathrm{O}(\mathrm{p}=0.12)$. The $6 \mathrm{MWT}$ in the pre was $386 \pm 43$ in the IG and $398 \pm 56$ meters $(\mathrm{p}=0.65)$. In the CG, at discharge they covered $285 \pm 51$ meters in the IG and $288 \pm 45$ in the CG $(\mathrm{p}=0.34)$. There were no differences between the variables of the groups. Conclusion: It is concluded that the IMT performed in the postoperative period did not show significance on the variables muscle strength and functional capacity in patients who were at high risk of pulmonary complications.
\end{abstract}

\section{INTRODUCTION}

The pathologies involving the cardiovascular system are constant and increasing, in addition, they represent a high rate of death worldwide, reaching up to $25 \%$ in developed countries. The number of cardiac surgeries, mainly myocardial revascularization (MRI), has been increasing worldwide. Approximately 45,000 surgeries in this category are performed in the United States annually ${ }^{1,2}$.

Despite being frequently performed, CABG is still a high-risk procedure, from its completion to the postoperative period, due to the impairments caused by this procedure ${ }^{3,4}$. There is evidence to prove that CABG has a great influence on the weakness of these patients, from the reduction of pulmonary capacities to functional ones. In addition to the immobilization caused by the postoperative period, the reduction of peripheral muscle strength is another factor that influences the decrease in functional capacity, where it is assessed through the six-minute walk test ${ }^{5-7}$.

These conditions when added to predisposing factors such as: age, sex, obesity, smoking, diabetes mellitus 
(DM), arterial hypertension (SAH) and dyslipidemia (DLP), have a greater influence on postoperative complications, the main ones being: atelectasis, stroke pleural, pneumonia and pneumothorax, conditions that can be minimized through early mobilization and Inspiratory Muscle Training (IMT) ${ }^{8-10}$.

IMT has shown its efficacy and benefits in patients who undergo MRI surgeries both pre- and postoperatively, contributing to the prevention of losses in pulmonary and functional aspects ${ }^{11}$. However, there is a knowledge gap regarding disrespect about the use of IMT on lung function and functionality in patients who present risk factors for the development of postoperative pulmonary complications (PPC).

The aim of this study is to assess the impact of IMT on the functional capacity of patients undergoing CABG who have risk factors for pulmonary complications.

\section{METHODS}

This is a randomized clinical trial, performed on patients undergoing myocardial revascularization surgery. Data were collected at the Instituto Nobre de Cardiologia (INCARDIO), Feira de Santana - Bahia, from January to October 2018. Our study was submitted and approved by the Ethics and Research Committee of Faculdade Nobre de Feira de Santana, obtaining the opinion number 2,366,995. All participants signed an informed consent form.

\section{Inclusion and exclusion criteria}

The inclusion criteria for this work were: individuals older than 60 years, smokers, patients with chronic obstructive pulmonary disease (COPD) confirmed by clinical and spirometric exam and body mass index (BMI) greater than $27 \mathrm{~kg} / \mathrm{m} 2$, both genders and undergoing myocardial revascularization.

The exclusion criteria were: death, surgical reintervention, use of intra-aortic balloon, heart valve diseases, pneumopathies, patients with difficulty in understanding the imposed techniques, presenting hemodynamic instability during the assessment or execution of the IMT and physical limitation compromising the assessment of functional capacity.

\section{Study protocol}

In the pre-surgical period, the following variables were individually assessed in all patients: pulmonary function, ventilatory muscle strength, peak flow and functional capacity. The pulmonary function variables evaluated were vital capacity and peak expiratory flow. Muscle strength through maximum inspiratory pressure (MIP) and maximum expiratory pressure (MEP). And, for functional capacity, the 6-minute walk test (6MWT) was used.

As predicted, the day after the evaluation, the patients were referred to the operating room and, after the procedure, they were admitted to the Intensive Care Unit (ICU). On the second postoperative day (POD), patients were randomized by simple drawing: there were both options on folded papers and someone working at the unit was asked to take a paper to draw.

Thus, these patients were assigned to one of the groups, namely: inspiratory muscle training group (IG) and the control group (CG). The IG had the MIP evaluated and $40 \%$ of the value found in each patient was used. To offer the load, the PowerBreathe device (PowerBreathe Medical Classic) was used. All patients included in this study performed 30 daily repetitions, divided into two shifts, 15 in the morning and 15 in the afternoon, every day until hospital discharge, in which all variables were reevaluated.

The CG evaluated in the preoperative period was submitted to interventions, such as: breathing exercises, ambulation, cycle ergometry, changes in decubitus, active or active assisted kinesiotherapy that were already performed according to the hospital protocol. All patients were reassessed at discharge. It is worth mentioning that all evaluations were made by a blind examiner.

Verification of Ventilatory Muscle Strength 
The preoperative assessment of inspiratory muscle strength (Maximum Inspiratory Pressure (MIP)) was performed using an Indumed( $\mathbb{R}$ analog manovacuometer. During the evaluation, the patient was asked for a maximum expiration until the residual volume and, then, a maximum and prolonged inspiration until his Total Pulmonary Capacity. This test was carried out with the unidirectional valve method, allowing flow through a millimeter orifice in order to exclude the action of the buccinator muscle, the test will be repeated three times, using the highest value obtained as long as it was not the last.

Another aspect evaluated was the expiratory muscle strength (Maximum Expiratory Pressure (MEP)), which was measured with the same device, but changing the way it was performed. Initially, the patient was instructed to perform a maximum inspiration until reaching his total pulmonary capacity. Then, after the mask was positioned, a maximum expiration was requested, until reaching the residual capacity, following the same rule of MIP, using the highest value, except when this is the last ${ }^{12}$.

\section{Pulmonary Function Measurement}

Vital capacity was one of the variables collected, for its evaluation an Analog Ventilometer (Ferraris - Mark 8 Wright Respirometer, Louisville, CO, USA) was used. The device was connected to a face mask (in the expiratory branch) and the patient was guided over all the phases of the test, then it was unlocked, zeroed and, soon after, the face mask was positioned on the patient's face, performing a deep inspiration until the total pulmonary capacity. Subsequently, a long and gradual exhalation was performed until reaching the residual volume. After the instructions and the procedure, the ventilometer was temporarily locked and the result was observed and recorded. The test was repeated three times and the highest value was taken into account ${ }^{13}$.

Peak expiratory flow was another variable, assessed using the Mini Wright $\AA$ peak flow. During the evaluation, the patient was seated, with his head in neutral and using a nose clip - preventing air from escaping through the nostrils. Then, the patient was asked to take a deep breath, until the total pulmonary capacity, and then a forced exhalation was performed with the mouth on the device. After three measurements were made, the highest value was chosen, with no difference greater than 40 liters between measurements ${ }^{13}$.

\section{Functional Capacity Assessment}

The Six-Minute Walk test (6MWT) is a simple and low-cost assessment, which was performed following the recommendations of the American Thoracic Society (ATS). The 6MWT is performed on a flat surface, free of obstacles and with 30 meters. Before performing the test, the patient rests for 10 minutes, during this period, some data such as blood pressure (using the Premium Aneroid sphygmomanometer and Littmann 3M stethoscope), pulse oximetry (Pulse Oximeter - Rossmax), level dyspnea (Borg scale), heart rate (assessed by palpation of the radial artery and counting over a period of one minute) and respiratory (assessment by checking the respiratory incursion over the period of one minute).

Therefore, the patient was asked to walk as quickly as possible, for six minutes, the therapist must observe the patient's physiological responses and finally the distance covered according to the variables of each individual. This test is currently considered the best indicator for measuring functional capacity, in addition to considering the risk of returning to the hospital, it also observes the patient's performance when related to activities of daily living ${ }^{13}$.

During the entire 6MWT process, the patient was monitored, the test could be interrupted if found: heart rate below $20 \%$ of the baseline, increased systolic and / or diastolic blood pressure greater than $30 \%$ of the base, increased respiratory rate above 25 incursions per minute and peripheral oxygen saturation $<90 \%$.

\section{Statistical analysis}

The analysis was performed using the SPSS 20.0 program. Normality was assessed using the Shapiro-Wilks test. The variables were expressed as means and standard deviations. Chi-square was used to compare categorical variables. To compare values between groups, the independent Student's T test was used and the intra-group comparison was the paired Student's T test. It was considered as significant when $\mathrm{p}<0.05$. 
The delta value ([?]) was found by subtracting the preoperative value from the value found on the day of discharge.

\section{RESULTS}

During the study period 38 patients were hospitalized, of these nine were excluded, two of whom were due to combined surgery, six lung diseases and one due to physical limitations, leaving 29 individuals as shown in figure 1 . The prevalence was male with 18 patients $(62 \%)$, the mean age was $67+-4$ years, the BMI was $31.5+-2.5 \mathrm{~kg} / \mathrm{m} 2$ and the prevalent comorbidity was sedentary lifestyle with 21 patients $(72.4 \%)$. Regarding the surgical and clinical variables, all values are shown in table 1.

Regarding muscle strength, a decrease of $22 \%$ was observed in the MIP in the IG and in the $28 \%$ in the CG $(\mathrm{p}=0.12)$. In MEP, IG decreased by $26 \%$ and CG by $34 \%(\mathrm{p}=0.21)$. These variables were not significant and are represented with absolute values in Table 2.

The 6-min walk test was performed before and after the operation and when compared, the IG had a drop of $26 \%$ in relation to the distance covered in meters and the CG $27 \%(\mathrm{p}=0.21)$. Table 3 shows the information regarding functional capacity.

\section{DISCUSSION}

In the data analysis of the present study, it was observed that the inspiratory muscle training protocol, applied to patients at high risk of pulmonary complications, did not show any significant difference in relation to the variables of MIP, MEP and 6MWT in patients undergoing coronary artery bypass grafting.

Pulmonary function is one of the factors that presents changes in the postoperative period, Barros et al. showed that there is a significant reduction in ventilatory capacity and muscle strength in patients who underwent MR. Still, in this same study, it was observed that the IMT helped to restore the overlapping values evaluated in the preoperative period ${ }^{14}$. Similar results have been reported by Matheus et al. who demonstrated the effectiveness of IMT in recovering the values of tidal volume and vital capacity found in the pre-surgical ${ }^{15}$. In the present study, a reduction in values was also observed when comparing pre and postoperative in patients who presented risk factors that may generate PPC, however these numbers were not significant, both in the control group and in the intervention group.

Possible justifications may be related to the reduction of ventilatory mechanics, among which are the presence of drains and pain. Sternotomy is an aggressive procedure, which causes several dysfunctions and can cause pain until the fifth postoperative day ${ }^{16}$. This symptom is common and can cause restriction of chest expansion, causing hypoventilation and making IMT difficult.

Cordeiro et al. presented different results and a similar population in relation to the current study. The researchers investigated the influence of IMT on inspiratory muscle strength and functional capacity. They observed a significant reduction in MIP in both groups, but the intervention group had a higher value for this variable at hospital discharge, compared to the control group. An analysis of functional capacity showed an improvement in the $6 \mathrm{MWT}$ when comparing the IMT group to the control group ${ }^{3}$.

This result is probably related to the researched sample, which was larger when compared to the present study and included other types of surgery besides MRI, considered to be large. Therefore, the greater number of patients, the different inclusion criteria, and, especially, the risk factors present increase the likelihood of PPC, heterogeneous characteristics among the studied samples.

With a more prolonged IMT protocol and with progressive load increase, Praveen et al. they also investigated the effectiveness of IMT in patients who underwent CABG and showed a reduction in MIP in both groups both in the short term and over time in the postoperative period. The group that underwent intervention presented approximate values preoperatively, however some of them increased significantly at maximum inspiratory strength ${ }^{17}$.

In the training mentioned above, patients were stimulated and instructed to perform IMT from the fourth 
to the nineteenth postoperative period, with a load that could increase according to the patient's resistance. Unlike the current research, in addition to extending the intervention period, the fact of starting IMT on the fourth postoperative day can reduce characteristics such as pain, analgesic effect and the patient's concern about sternotomy. Generally, these findings are still present in the second POD, making it possible to compromise load measurement and training ${ }^{17}$.

In this context, it is necessary to emphasize that the barriers found in the postoperative period are not limited to the restrictions of the surgical procedure. Predisposing risk factors such as: age, sex, obesity, smoking, diabetes mellitus, arterial hypertension and dyslipidemia are aggravating factors that guide the team about possible complications during or after surgery, directly affecting the recovery of functional and respiratory capacity, increasing the chance morbidity and mortality ${ }^{18}$.

A study carried out Feltrim et al. sustained the benefit of IMT in high-risk patients with factors similar to the current study and demonstrated a reduction in pulmonary complications of greater impact, acting in a protective way, training improved the strength and resistance of the respiratory musculature ${ }^{8}$. IMT could probably decrease complications in the studied population, but the functional capacity is dependent on other conditions, not influencing the postoperative risk factors.

Nery et al. also used the 6MWT to assess functional capacity in individuals who underwent MRI surgery. The patients were divided into a sedentary and active group, who performed physical exercises and IMT. In the evaluation, after two years, patients who performed exercises associated with IMT showed less loss in functional capacity, showing significant improvement in the distance covered in relation to sedentary patients. This result may be associated with IMT for a prolonged period and the performance of exercise ${ }^{19}$.

Physical exercises are beneficial in wide aspects, considerably improve hemodynamics and cardiac output. Demonstrating more effective recovery of the myocardium where they consequently improve muscle fatigue, caused by the decrease in blood distribution, reducing the feeling of tiredness. When associated with IMT, patients effectively improve functional capacity and optimize pulmonary capacity ${ }^{20-22}$.

In relation to the $6 \mathrm{MWT}$, researchers evaluated the functional capacity in patients with risk factors who underwent CABG and noticed an improvement in the distance covered in older individuals, aged over 75 years, with greater benefit in relation to the distance covered, unlike the present study that had an average age of 67 years ${ }^{23,24}$. This is one of the inconstant factors, however it is understood that when there is an imminent risk in elective surgeries, it is important to carry out preventive interventions, such as preoperative training, which can train the muscles and minimize impacts on MIP and MEP and probably in the distance covered in the postoperative ${ }^{8}$.

Our work has some limitations, such as the sample size and the absence of patients' effort evaluation (BORG scale), which could have been used to gradually increase the load. The different study protocols used by the authors are also characterized as a work limitation.

\section{CONCLUSION}

Based on the results presented, we can conclude that the IMT performed in the postoperative period did not show any significant influence in relation to the risk factors presented, when related to muscle strength and functional capacity.

However, taking into account the little representativeness of the studied sample and the absence of authors who address the risk of complications and functional capacity in this population, further research is needed to obtain better results.

\section{REFERENCES}

1. Calles ACN, Lira JPF, Granja KSB, Medeiro JD, Farias AR, Cavalcanti RC. Pulmonary complications in patients undergoing coronary artery bypass grafting at a hospital in Maceio, Brazil. Fisioter Mov; 2016; $29(4): 661-7$. 
2. Santos VM, Neto EN, Silva Nazario MP, Bittencourt WS, Salicio MA, Nasrala MLS. Capacidade Funcional e Forca Muscular de Pacientes Submetidos a Revascularizacao do Miocardio. J Health Sci; 2018; 20(1):45-9.

3. Cordeiro ALL, Melo TA, Neves D, Luna J, Esquivel MS, Guimaraes ARF et al. Inspiratory muscle training and functional capacity in patients undergoing cardiac surgery. Braz J Cardiovasc Surg; 2016; $31(2): 140-4$.

4. Szylinska A, Listewnik M, Rotter I, Ryl A, Kotfis K, Mokrzycki, K et al. The Efficacy of Inpatient vs. Home-Based Physiotherapy Following Coronary Artery Bypass Grafting. Int. J. Environ. Res. Public Health; 2018; 15(2):2-11.

5. Morales-Blanhir JE, Vidal CDP, Romero MJR, Castro MMG, Villegas AL, Zamboni M et al. Six-minute walk test: a valuable tool for assessing pulmonary impairment. J Bras Pneumol; 2011; 37(1):110-7.

6. Carey EJ, Steidley DE, Aqel BA, Byrne TJ, Mekeel KL, Rakela J. et al. Six minute walk distance predicts mortality in liver transplant candidates. Liver Transplantation; 2010; 16:1373-8.

7.Soman A, Mundyat G, Kumar D, Santhakumar H. Does body mass index influence pulmonary function test values and functional exercise capacity after chest physiotherapy following coronary artery bypass graft. Indian J Thorac Cardiovasc Surg; 2017; 34(2):116-124.

8. Feltrim MIZ, Jatene FB, Bernardo WM. Em pacientes de alto risco, submetidos a revascularizacao do miocardio, a fisioterapia respiratoria pre-operatoria previne as complicacoes pulmonares? Rev Assoc Med Bras; 2007; 53(1):1-12.

9. Nascimento PV, Jesus APS, Cunha EN, Rosario NCS, Guimaraes ACG. Cardiovascular risk factors in patients submitted to coronary bypass graft surgery. 2016. Rev enferm UFPE on line; 2016; 10(2):1007-15.

10. Steffens E, Dallazen F, Sartori C, Chiapinotto S, Battisti IDE, Winkelmann ER. Condicoes fisicofuncionais e qualidade de vida de pacientes no pre e pos- operatorio de cirurgia cardiaca. Revista Pesquisa em Fisioterapia; 2016; 6(4):422-429.

11. Kalil-Filho FA, Campos ACL, Tambara EM, Tome BKA, Trerml CJ, Kuretzki CH, et al. Physiotherapeutic approaches and the effects on inspiratory muscle force in patients with chronic obstructive pulmonary disease in the pre-operative preparation for abdominal surgical procedures. ABCD Arq Bras Cir Dig; 2019; $32(2): 1-4$.

12. Neder JA, Andreoni S, Lerario MC, Nery LE . References values for lung function tests. II. Maximal respiratory pressures and voluntary ventilation. Braz J Med Biol Res; 1999; 32(6): 719-27.

13. American Thoracic Society, European Respiratory Society. Statement on Respiratory Muscle Testing. Am J Respir Crit Care Med; 2002; 166:518-624.

14. Barros GF, Santos CS, Granado FB, Costa PT, Limaco RP, Gardenghi G. Respiratory muscle training in patients submitted to coronary arterial bypass graft. Rev Bras Cir Cardiovasc; 2010; 25(4): 483-490.

15. Matheus GB, Dragosavac D, Trevisan P, Costa CE, Lopes MM, Ribeiro GCA. Treinamento muscular melhora o volume corrente e a capacidade vital no pos-operatorio de revascularizacao do miocardio. Rev Bras Cir Cardiovasc; 2012; 27(3): 362-9.

16. Giacomazzi CM, Lagni VB, Monteiro MB. A dor pos-operatoria como contribuinte do prejuizo na funcao pulmonar em pacientes submetidos a cirurgia cardiaca. Braz J Cardiovasc Surg; 2006; 21(4): 386-392.

17. Praveen R, Swaminathan N, Praveen JS. Inspiratory Muscle Training is Effective in Improving Respiratory Muscle Functions in Patients who have Undergone Coronary Artery Bypass Graft. Fizjoterapia Polska; 2009; 9 (4): 285-292.

18. Katsura M, Kuriyama A, Takeshima T, Fukuhara S, Furukawa TA. Preoperative inspiratorymuscle training for postoperative pulmonary complications in adults undergoing cardiac and major abdominal surgery 
(Review). Database of Systematic Reviews; 2015: 10.

19. Nery RM, Martini MR, Vidor CR, Mahmud MI, Zanini M, Loureiro A, et al. Alteracoes na capacidade funcional de pacientes apos dois anos da cirurgia de revascularizacao do miocardio. Rev Bras Cir Cardiovasc; 2010; 25(2): 224-228.

20. De Castro RRT, Negrao CE, Stein R, Serra SM, Teixeira JAC, Carvalho T, et al. Diretriz de reabilitacao cardiaca. Arquivos Brasileiros de Cardiologia; 2005; 84(5): 431-440.

21. D. Laoutaris I, Dritsas A, L. Louca L. Adamopoulos S. Contribution of aerobic/resistance/ inspiratory muscle training to cardiopulmonary recovery in a recipient of a ventricular assist device: Off-pump evaluation. European Journal of Preventive Cardiology; 2018; 00(0): 1-3.

22. Dos Santos TD, Pereira SN, Portela LOC, Cardoso DM, Dal Lago P, Guarda NS, et al. Moderate-tohigh intensity inspiratory muscle training improves the effects of combined training on exercise capacity in patients after coronary artery bypass graft surgery: A randomized clinical trial. International Journal of Cardiology; 2018; 279: 40-46.

23. Macchi C, Fattirolli F, Molino Lova R, Conti AA, Luisi MLE, Intini R, et al. Early and Late Rehabilitation and Physical Training in Elderly Patients After Cardiac Surgery. Am. J. Phys. Med. Rehabil; 2007; 86 (10): 826-834.

24. Gomes Neto M,Martinez BP, Reis HFC, Carvalho VOC. Pre- and postoperative inspiratory muscle training in patients undergoing cardiac surgery: Systematic review and meta-analysis. Clinical Rehabilitation; 2016;31(4): 454-464.

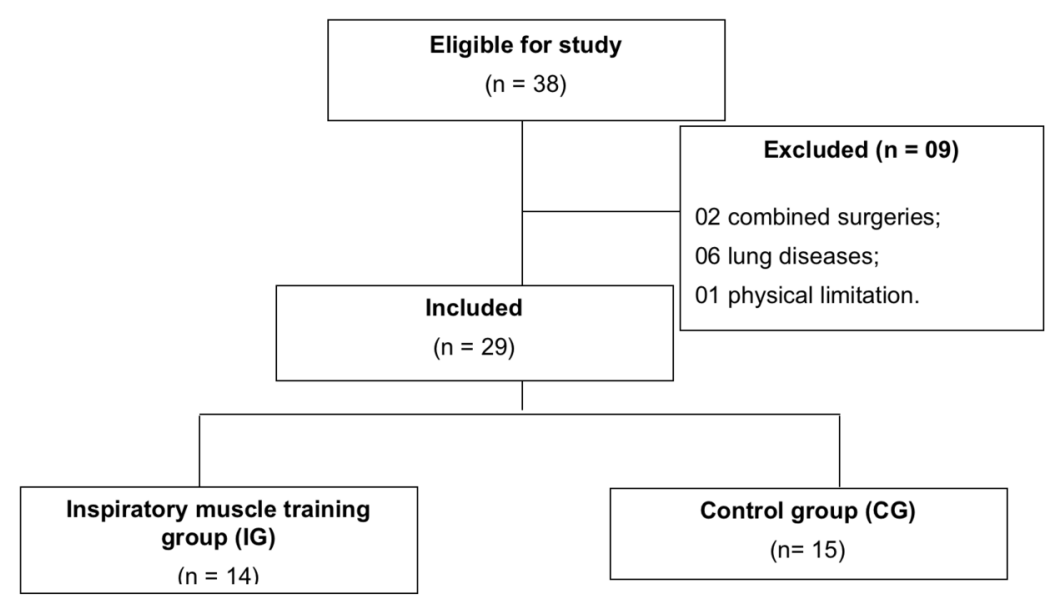

Figure 1: Steps for selecting the study subject.

Table 1. Clinical and surgical data of patients undergoing coronary artery bypass grafting.

\begin{tabular}{llll}
\hline Variables & IG $(\mathrm{n}-14)$ & CG $(\mathrm{n}-15)$ & $\mathrm{p}$ \\
\hline Gender Male Female & $9(64 \%) 5(36 \%)$ & $9(60 \%) 6(40 \%)$ & $0,31^{\mathrm{a}}$ \\
Age $($ years $)$ & $66 \pm 3$ & $68 \pm 4$ & $0,65^{\mathrm{b}}$ \\
BMI $\left(\mathrm{kg} / \mathrm{m}^{2}\right)$ & $32 \pm 2$ & $31 \pm 3$ & $0,42^{\mathrm{b}}$
\end{tabular}




\begin{tabular}{llll}
\hline Variables & IG $(\mathrm{n}-14)$ & CG $(\mathrm{n}-15)$ & $\mathrm{p}$ \\
\hline Comorbidities DM & $9(64 \%) 10(71 \%) 9$ & $9(60 \%) 9(60 \%) 10$ & $0,32^{\mathrm{a}} 0,64^{\mathrm{a}} 0,57^{\mathrm{a}} 0,34^{\mathrm{a}}$ \\
SAH DLP Sedentary & $(64 \%) 11(79 \%) 7$ & $(67 \%) 10(67 \%) 5$ & $0,24^{\mathrm{a}}$ \\
lifestyle AMI & $(50 \%)$ & $(33 \%)$ & \\
MV time (horas) & $6 \pm 2$ & $7 \pm 3$ & $0,23^{\mathrm{b}}$ \\
CPB time (min) & $96 \pm 12$ & $92 \pm 15$ & $0,23^{\mathrm{b}}$ \\
Number of grafts & $2,6 \pm 0,8$ & $2,5 \pm 0,6$ & $0,65^{\mathrm{b}}$ \\
\hline
\end{tabular}

a. Chi-square; b. Independent Student's T Test; CPB - Cardiopulmonary bypass; DLP - Dyslipidemia; DM - Diabetes Mellitus; SAH - Systemic Arterial Hypertension; AMI - Acute Myocardial Infarction; BMI - Body Mass Index; MV - Mechanical ventilation.

Table 2. Pulmonary Function and Ventilatory Muscle Strength preoperatively and at hospital discharge between groups.

\begin{tabular}{llll}
\hline Variables & IG $(\mathrm{n}-14)$ & $\mathrm{CG}(\mathrm{n}-15)$ & $\mathrm{p}^{\mathrm{a}}$ \\
\hline $\mathrm{MIP}\left(\mathrm{cmH}_{2} \mathrm{O}\right)$ & $103 \pm 1580 \pm 1423 \pm$ & $105 \pm 1775 \pm 1530 \pm$ & $0,350,560,12$ \\
Preoperative Discharge & 13 & 12 & \\
$\Delta$ & $82 \pm 1260 \pm 1122 \pm$ & $85 \pm 1356 \pm 1229 \pm$ & $0,420,560,21$ \\
MEP $\left(\mathrm{cmH}_{2} \mathrm{O}\right)$ & 11 & \\
Preoperative Discharge & 11 & & \\
$\Delta$ & & & \\
\hline
\end{tabular}

a. Independent Student's T Test; MEP - Maximum Expiratory Pressure; MIP - Maximum Inspiratory Pressure; $\Delta$ - Delta.

Table 3. Preoperative functional capacity and hospital discharge between groups.

\begin{tabular}{llll}
\hline Variables & IG $(\mathrm{n}-14)$ & CG $(\mathrm{n}-15)$ & $\mathrm{p}^{\mathrm{a}}$ \\
\hline 6MWT Preoperative & $386 \pm 43285 \pm 51101$ & $398 \pm 56288 \pm 45110$ & $0,65^{\mathrm{a}} 0,34^{\mathrm{a}} 0,21^{\mathrm{a}}$ \\
Discharge $\Delta$ & \pm 45 & \pm 46 & \\
\hline
\end{tabular}

a. Independent Student's T Test; 6MWT - Six-Minutes Walking Test; $\Delta$ - Delta 